\title{
Electrophysiological correlates of visual binding errors after bilateral parietal damage
}

Elena Pedrazzini ${ }^{1}$, Julia Fellrath ${ }^{1,2}$, Raphaël Thézé ${ }^{1}$ and Radek Ptak ${ }^{1,2,3}$

${ }^{1}$ Laboratory of Cognitive Neurorehabilitation, Faculty of Medicine, University of Geneva, Geneva, Switzerland

${ }^{2}$ Division of Neurorehabilitation, Geneva University Hospitals, Geneva, Switzerland

${ }^{3}$ Faculty of Psychology and Educational Sciences, University of Geneva, Geneva,

Switzerland

\section{Corresponding author address:}

Radek Ptak

Division of Neurorehabilitation, Geneva University Hospitals and

Faculty of Medicine, University of Geneva

26, Av. de Beau-Séjour, 1211 Geneva 14, Switzerland

Tel.: +41-22-382 35 24; Fax: +41-22-382 8338

radek.ptak@hcuge.ch 


\section{ABSTRACT}

Illusory conjunctions (e.g. the confusion between the shape of one stimulus with the color of another stimulus) are the most dramatic expression of binding failures in vision. Under brief exposure or when attention is diverted illusory conjunctions may be observed in healthy participants, but they only represent a real-life problem for patients with parietal damage. However, it is unclear whether such failures reflect the impairment of early or late stages of visual processing. Here, we examined the timecourse of visual processing using evoked potential measures in a patient with bilateral damage to the posterior parietal cortex presenting prominent binding failures. The patient was asked to identify colored letters that were briefly flashed to the left or right hemifield. When only one item was presented she adequately identified color or shape of left and right letters. In contrast, when presentation was bilateral she either identified the correct right shape-color combination and missed the item in the left hemifield (extinction) or combined incorrectly the right shape with the left color (illusory conjunction). Evoked potential analyses revealed a specific electrophysiological signature of illusory conjunctions, starting 105 ms after stimulus onset over the right frontal cortex. These findings indicate that binding errors reflect failures of early stages of attentional filtering relying on the integrity of the posterior parietal cortex.

Keywords: Visual attention; parietal lobe; binding; features; illusory conjunction.

During the initial stages of visual processing at the cortical level information is highly segregated across a multitude of areas specialized for shape, orientation, color or motion (Felleman and Van Essen, 1991, Reynolds and Desimone, 1999). Due to this specialization feature representations are distributed across distinct cortical regions of visual cortex. Where and when a unified and integrated representation of an object is elaborated by recombining distinct features is referred to as the binding problem (von der Malsburg, 1995, Treisman, 1996).

The existence of a binding problem in vision is exemplified by visual search studies. The search for a target characterized by a distinct feature (e.g. a single red letter among green letters) is very fast, effortless and independent of the number of distracters. In contrast, searching for a distinctive combination of features (e.g., a vertical red line among vertical green and horizontal red lines) generally leads to effortful search with response times that increase linearly with increasing numbers of distracters (Treisman and Gelade, 1980, Eckstein, 2011). Many basic stimulus attributes such as color, orientation or shape can be processed preattentively, and often in parallel in early vision (Treisman, 1998). However, unless attention is directed to them they only appear as loose collections of features rather 
than as coherent visual objects (Wolfe and Bennett, 1997). In contrast, attentive vision has limited capacity, but allows localizing single features and feature combinations. Though under some experimental conditions (in particular if features are rendered salient) the search for feature combinations may be effortless (Wolfe, 1994), many authors agree that the perception of coherent, spatially localized objects requires focal attention and that capacity limitations lead to binding failures (Treisman, 1998, Cave and Bichot, 1999, Wolfe and Cave, 1999). Thus, at the phenomenological level preattentive vision may signal the presence of redness and curves, while attentive vision may identify a red apple (Billock and Tsou, 2004). Functional imaging studies have shown that attentive processing of feature combinations strongly activates the superior parietal cortex (Corbetta et al., 1995, Shafritz et al., 2002, Egner et al., 2008). This finding is consistent with experimental studies on rare patients with bilateral parietal damage showing deficits in visual search, localization and binding of different stimulus features (Rafal, 1997, Robertson, 2003). When shown brief displays these patients may erroneously report illusory conjunctions that is, feature combinations that were not present in the display. Illusory conjunctions have been observed for combinations of shape and color, shape and size, or color and motion (Friedman-Hill et al., 1995, Bernstein and Robertson, 1998, Humphreys et al., 2000, Valenza et al., 2004).

Though under very brief presentation conditions and especially in peripheral vision illusory conjunctions may be provoked in healthy observers (Treisman and Schmidt, 1982, Billock and Tsou, 2004), they only represent a real-life problem in patients with parietal damage (Cohen and Rafal, 1991, Friedman-Hill et al., 1995). The binding deficit in these patients is secondary to a severe impairment of explicit spatial localization (Cinel and Humphreys, 2006), and was therefore hypothesized to reflect the failure of a late stage of visual processing (Robertson et al., 1997). This proposal is in agreement with the feature integration theory, which states that single features are separately processed in parallel in preattentive feature maps and then integrated at a later stage where the spatial positions of distinct features become available (Treisman and Gelade, 1980, Treisman, 1998). In addition, early versions of feature integration theory proposed that spatial attention is necessary for feature binding and acts as 'glue', binding features that are within its spotlight into a coherent object (Quinlan, 2003). Support for two-stage models such as feature integration theory has mainly come from visual search studies, and in particular from the observation that search for a target characterized by a single salient feature is fast and largely independent of the number of distracters while search for a feature conjunction is slow and effortful (Treisman and Gormican, 1988, Quinlan, 2003). However, this experimental finding contrasts with the relative ease and velocity with whitch healthy observers perceive complex feature combinations characterizing everyday objects. In addition, visual search for a single feature may be rendered difficult and effortful when the target is only slightly distinct from the distracters, while under some conditions feature 
combinations may be detected in the absence of focused attention (Duncan and Humphreys, 1989, Holcombe and Cavanagh, 2001, Wolfe and Horowitz, 2004). In addition, though a role of attentional limitation by brief exposures, crowding or diverted attention has been considered as crucial for the occurrence of illusory conjunctions (Wolfe and Cave, 1999), some studies obtained feature integration errors with long durations and without diverting attention (Prinzmetal et al., 1995). Thus, behavioral studies do not make it clear whether illusory conjunctions result from the failure of early or late perceptual processes. A possible alternative is to study electrophysiological correlates of visual binding errors by measuring event-related potentials. However, while there is a tradition of studying visual search performance with event-related potential (ERP) methods (Luck et al., 1993, Luck and Hillyard, 1994, Leonards et al., 2003, Hickey et al., 2009, Eimer and Kiss, 2010), these studies focused on attentional mechanisms implicated in serial or parallel search rather than on the attentional failures leading to visual binding errors.

Here, we studied a rare patient with bilateral damage to the posterior parietal cortex exhibiting prominent binding errors when shown simple displays containing colored letters. We took advantage of the excellent temporal resolution of ERP measures to investigate whether binding in our patient depended on early (preattentive) feature combination processes, or whether a later process requiring focused attention is necessary. We found that illusory conjunctions were associated with a specific electrophysiological signature starting 90 ms following stimulus presentation, indicating that visual binding relies on early selection processes.

Insert Figure 1 about here

\section{EXPERIMENTAL PROCEDURES}

\section{Participant}

EB, a right-handed 74 year-old woman, suffered from two consecutive strokes that left her with a combination of symptoms characteristic of Bálint's syndrome: inability to perceive more than one object at a time (simultanagnosia), optic ataxia and ocular apraxia (Bálint, 1909, Rafal, 1997, Ptak and Müri, 2013). High-resolution structural MRI performed three years following the second stroke revealed chronic necrotic changes affecting mainly the supramarginal and postcentral gyrus of the right hemisphere and the angular, supramarginal and superior occipital gyrus of the left hemisphere (Figure 1A).

At the time of this study the patient had recovered from ocular apraxia and optic ataxia up to a point allowing her to gain independence in some daily activities, but continued to show severe deficits of spatial attention. Her visual fields were preserved on confrontation testing, 
and she could identify simple shapes, colors, or everyday objects, but frequently made visual errors suggesting that she failed to explore an object in its entirety (e.g., she would confound a match with a crayon). Visual extinction was complete on confrontation testing, mostly affecting the left side. Table 1 shows results of neuropsychological testing of EB. EB showed moderate to severe impairments in almost all visual and spatial tests. There were no signs of spatial neglect in everyday behavior or in cancellation (Gauthier et al., 1989), but she missed many targets in a cancellation task. Reading of single words was impossible (e.g., she only identified the letter $\mathrm{N}$ in the French word lune-moon), though she read most single letters correctly. The patient was lost when asked to indicate the position, the orientation or the spatial relation between objects, and she was unable to find her way in a new environment. Simultanagnosia and severe spatial confusion were evident when EB attempted to enumerate or to name several objects placed in front of her on the table. Though she named one of the items she was neither able to localize or to seize it nor to indicate which other objects were present. In everyday life an additional problem became evident: EB was unable to provide the color of an object that she had correctly identified (e.g., a blue pen), often indicating the color of one of nearby objects. EB gave written informed consent to examine these visual confusions in a systematic study.

Insert Table 1 about here

\section{Stimuli and procedure}

Spatial extinction and feature binding errors were examined with briefly presented, white or colored letters drawn from the set A, B, F, H, L, P, T and $U$ and were presented in 110-point Arial font on black background. The stimuli were presented on a 20 " flat screen with a resolution of $1024 \times 768$, at a distance of $57 \mathrm{~cm}$ from the patient. Letter size was $4.2 \times 3.4$ degrees and their inner border was at a distance of 7 degrees from the central fixation cross (Figure 1B). In all experiments a trial started with the appearance of a white cross in the center of the screen. Presentation was under control of the experimenter who verified stable fixation before pressing the mouse button whereupon one or two letters appeared for $150 \mathrm{~ms}$ on the screen. The patient was notified that there could be one or two letters on the screen. In Experiment 1 white letters were shown and EB was asked to identify each letter and to indicate by pointing whether it was presented left or right of fixation. There were 48 unilateral left or right and 104 bilateral trials presented in two blocks. Experiment 2 was similar to Experiment 1 except that single $(n=56)$ or double $(n=112)$ stimuli were presented in the upper or lower visual field. In Experiment 3a letters were colored red (RGB: 255,0,0), blue 
$(0,0,255)$, green $(0,255,0)$ or yellow $(255,255,0)$. For bilateral stimulations all combinations of letters and colors could occur except for the repetition of two same letters or colors. EB was asked to indicate letter shape and color, and to point for each identified feature to the hemifield where it was presented. There were 56 unilateral left or right and 112 bilateral presentations. Experiment 3b was a replication of Experiment 3a, but with simultaneous EEG recording. In addition, only the red, blue and yellow colors were used as EB found it difficult to differentiate blue and green in Experiment 3a. Also, in order to limit EEG movement artifacts we did not ask EB to indicate the location of letters, but merely to name them. There were 196 unilateral left or right and 426 bilateral trials, distributed across seven blocks and three EEG sessions.

\section{EEG acquisition, processing and analysis}

EEG was acquired through a 128-channel system with electrodes positioned according to the extended 10/20 system (Biosemi V.O.F, Amsterdam, Netherlands), with a sampling rate of $512 \mathrm{~Hz}$. EEG data pre-processing and analyses were performed using Cartool software (Brunet et al., 2011). Data were band-pass filtered $(0.1-100 \mathrm{~Hz})$ and recalculated against the average reference. Trials with artifacts were excluded by applying an automatic rejection criterion of $+/-100 \mathrm{mV}$ and additionally by visual inspection for blinks, eye movements and muscular contractions. Channels presenting extended artifacts were excluded from analysis and interpolated from adjacent electrodes via 3D spline interpolation $(<5 \%$ interpolated electrodes; Perrin et al., 1987). Data were baseline-corrected by subtracting each block with the average EEG registered within the $100 \mathrm{~ms}$ pre-stimulus period and artifact-free epochs were averaged across the time period $-100 \mathrm{~ms}$ before to $400 \mathrm{~ms}$ after stimulus presentation and for each of the seven experimental blocks separately.

The behavioral results showed that while the patient adequately reported unilateral left or right items, she consistently perceived only one of two items in bilateral presentations (see Results section). Moreover, on some bilateral trials she reported the shape and color of the right letter (extinction trials), while on other trials she reported the color of the left item and the shape of the right item (conjunction trials). Since we were interested in the electrophysiological correlates of illusory conjunctions, the relevant comparison was between conjunction and extinction trials, as in both types of trials the displays were identical while the subjective experience of the patient differed. In order to control for deviations from normality in the data and to correct the family-wise error rate in multiple comparisons, we performed cluster-level statistics, followed by permutation tests (Maris and Oostenveld, 2007). We analyzed the data by first identifying significant clusters $(p<.05)$ of electrodes and then performed nonparametric permutation tests ( $N=1000$ permutations) to determine the likelihood with which each cluster explained the data. This analysis was performed using the FieldTrip toolbox implemented in Matlab (Oostenveld et al., 2011). 
Insert Figure 2 about here

\section{RESULTS}

\section{Behavioral results}

Figure 2A shows EB's performance with horizontally (Experiment 1) and vertically (Experiment 2) presented letters. We distinguished between responses in which EB indicated being aware that 'something' was presented on the screen (detection) from instances when she could clearly identify the letter. In horizontal trials performance for single left and right items was comparable (detection: $X^{2}=.26$; identification: $X^{2}=1.58$ ). In double trials EB showed complete extinction: she was never able to detect the presence of two items. Extinction was observed in $83.6 \%$ trials for the left and in $15.4 \%$ trials for the right item. Right items were detected $\left(X^{2}=2.11\right)$ or identified $\left(X^{2}=1.53\right)$ with similar accuracy on single or double trials, while performance was impaired for left items under double compared to single presentation (detection: $X^{2}=88.81, \mathrm{p}<.00001$; identification: $X^{2}=60.14, \mathrm{p}<.00001$ ). Finally, under double presentation right items were detected $\left(X^{2}=94.24, \mathrm{p}<.00001\right)$ and identified $\left(X^{2}=88.42, \mathrm{p}<.00001\right)$ better than left items.

In vertical presentations single letters presented in the upper or lower hemifield were detected $\left(X^{2}=.11\right)$ and identified $\left(X^{2}=.35\right)$ comparably well. In contrast, EB only once indicated seeing two items in double displays, exhibiting extinction either for the upper (21.6\% trials) or the lower (78.4\% trials) item. Performance for upper items in single or double displays was comparable for detection $\left(X^{2}=.90\right)$ and identification $\left(X^{2}=3.26\right)$, while lower items were processed less well in double than single displays (detection: $\chi^{2}=69.04, p$ $<.00001$; identification: $\left.X^{2}=68.56, p<.00001\right)$. Also, double presentation had a stronger effect on lower than upper hemifield items (detection: $\chi^{2}=95.65, p<.00001$; identification: $\left.x^{2}=80.26, \mathrm{p}<.00001\right)$.

These findings show that EB perceived correctly single letters, but under conditions of competition almost invariably showed extinction mainly for left and lower hemifield items. An additional deficit became apparent when she attempted to localize single stimuli (Figure 2B). With horizontal displays her performance was always at chance level except for localization of single right-sided items $\left(X^{2}=21.58, \mathrm{p}<.00001\right)$. In vertical displays she showed above chance performance only for letters presented in the upper visual field (single: $\chi^{2}=9.08, p<$ .01 ; double: $\left.x^{2}=19.99, \mathrm{p}<.00001\right)$. 
Identification of colored letters was very similar in Experiments $3 \mathrm{a}$ and $3 \mathrm{~b}$ and results were therefore pooled for analysis (Figure 3A). Under single presentation EB identified the combination of shape and color $\left(X^{2}=16.51, \mathrm{p}<.0001\right)$ and shape alone $\left(X^{2}=45.35, \mathrm{p}<\right.$ $.00001)$ better for right than left items. In contrast, her identification of color was equivalent for the left and right visual field $\left(X^{2}=.2\right)$. Under double presentation she showed significant decrements of performance for correct identification of shape, color and the conjunction of both features, and for left or right targets (all $x^{2}>10.31, p<.01$ ). However, while extinction of letter shape compared to color was greater for letters presented in the left hemifield $\left(X^{2}=\right.$ $142.01, \mathrm{p}<.00001)$, extinction of letter color was more important than extinction of shape for items in the right hemifield $\left(X^{2}=125.72, \mathrm{p}<.00001\right)$.

Insert Figure 3 about here

When asked to localize letters and colors (Experiment 3a) the patient made mainly errors for the color of the left letter, which she wrongly combined with the right letter shape (Figure 3B). These illusory conjunctions were nearly as frequent (31.5\% of all double trials) as the trials on which EB identified both features of the right letter correctly (43.2\%). The other possible illusory conjunctions (left letter shape with right letter color) occurred only rarely (1.4\%). Importantly, EB's tendency to make illusory conjunctions did not reflect random choice of color. Out of all instances when she identified the shape of the right letter, she ascribed to it the left color significantly more often than predicted by chance $\left(\mathcal{X}^{2}=37.82, \mathrm{p}<\right.$ $.00001)$. Thus, EB did not simply guess but perceived a spatially separated shape and color as two features of the same object.

Insert Figure 4 about here

\section{EEG results}

Figure 4 shows the configuration of electric fields of ERPs in three different time windows, related to different stages of information processing. The scalp topography of unilateral trials was characterized by a contralateral positivity in the early time window. Interestingly, the scalp topographies for bilateral extinction and conjunction trials strongly resembled the topography of unilateral left trials, although in both conditions the patient did not report the presence of the left stimulus. All four conditions resulted in a symmetrical voltage pattern at the scalp in the late time window. 
Figure 5 shows the results of nonparametric permutation tests comparing ERPs of a right frontal cluster of electrodes generated by illusory conjunctions against extinction trials. Note that in both types of trials EB was convinced to see a single colored letter, but that in conjunction trials she attributed the left color to the right item. Since the displays in both conditions were identical and only the subjective perception of the patient differed this comparison reveals the specific electrophysiological signature of illusory conjunctions. The comparison only identified a cluster of electrodes lying over the right frontal cortex which differed significantly between conditions in the time period 105-160 ms. Figure 6 depicts the ERP pattern for four electrodes belonging to this cluster (corresponding approximately to electrodes Fc4, Fc6, F4 and F6 in the international 10-20 electrode system) as compared to four left anterior (Fc3, Fc5, F3 and F5), right posterior (Cp4, Cp6, P4 and P6) and left posterior (Cp3, Cp5, P3 and P5) electrodes selected at symmetrical electrode sites. The figure shows that illusory conjunctions were associated with distinctive early electrophysiological differences between 105-160 ms at anterior right electrodes.

Insert Figures 5 and 6 about here

\section{DISCUSSION}

Integration of different parts of an object is crucial for the perception of a coherent environment, where shapes, colors and other properties are bound correctly to their respective locations. Cognitive models propose that binding is subsequent to the early elaboration of feature representations, and thus reflects a late stage of visual processing (Wolfe, 1994, Treisman, 1996). The present findings indicate that binding errors of patient EB reflect the failure of an early, spatially specific selection process.

Previous neuropsychological studies and the present report indicate that illusory conjunctions result from the combination of two deficits: a severe restriction of visual attention and impaired spatial localization. The findings of Experiment 1 and 2 show that EB indeed suffered from a severe attentional restriction that became evident when two stimuli competed for awareness. Under this condition EB showed complete extinction and consistently reported one stimulus only. The second deficit of EB was her difficulty to indicate the location of stimuli. She made many localization errors for single stimuli and her performance was random when two items were presented. Thus, under conditions of competition EB consciously perceived only one shape, but did not know where it was presented. This combination of attentional restriction and impaired localization appears to be necessary for the occurrence of illusory conjunctions. Interestingly, nearly all illusory conjunctions of EB were characterized by the displacement of the left color to the right. This finding has 
important implications for feature selection and binding. Psychophysical theories have proposed that what we term spatial attention is the result of a capacity limitation that restricts processing to relevant objects, binds their features into a coherent whole and makes them accessible to consciousness (Treisman, 1996, Wolfe and Bennett, 1997). In healthy subjects random illusory conjunctions can be observed when capacity limitations are induced using brief exposures, through fast succession of stimuli or by adding a secondary task (Treisman and Schmidt, 1982, Prinzmetal et al., 1995). However, EB's illusory conjunctions showed a systematic relationship, suggesting that attentional capacity was not only severely restricted, but also had different spatial selectivity for shape and color. Her attentional bottleneck only allowed selection of two, relatively simple features to be bound into one explicitly localizable object. Her pattern of performance can be explained by assuming competitive interactions between spatially separated features at each processing level (Desimone and Duncan, 1995, Reynolds and Desimone, 1999, Ptak and Fellrath, 2013). EB's performance under double presentation supports this assumption. She reported the right shape on the majority of trials, indicating that competition for shape was strongly biased in favor of the item presented in the right hemifield. However, for color competition was much more balanced; therefore, either the right or the left color may have passed the bottleneck and become bound to the rightsided shape.

Our ERP findings are in agreement with this interpretation. A previous EEG study with healthy participants suggested that illusory conjunctions are associated with smaller amplitudes in the $\mathrm{N} 1$ time range (>150 ms) at posterior electrodes (Mitko et al., 2015). We did not find ERP differences at posterior electrodes, which may be explained by the fact that these electrodes were located above damaged brain tissue producing abnormal or noisy electrophysiological activity. In contrast, right anterior electrodes recorded a distinct and systematic pattern of ERPs associated with illusory conjunctions between 105-160 ms. During this period the amplitude of ERPs to illusory conjunctions was increased relative to extinction trials. This ERP difference could not be attributed to purely bottom-up sensory processes, since in conjunction and extinction trials the visual stimuli were entirely comparable (two colored letters). This early time period therefore represents a specific electrophysiological correlate of the cognitive processes associated with illusory conjunctions.

How could such an early time window relate to cognitive processes underlying feature binding? Previous studies have shown that the earliest ERP correlates of visual awareness occur approximately 200 ms after stimulus onset (Koivisto and Revonsuo, 2010), and in parietal patients explicit awareness of an object suggests a late stage of visual processing (Robertson et al., 1997, Humphreys et al., 2000, Ptak and Schnider, 2005). The second temporal window identified in the topographic analysis (200-300 ms) might therefore reflect the time-point at which the representation of a colored letter as a coherent object becomes 
accessible to EB's consciousness. This interpretation is in agreement with the fact that in all experimental conditions the ERP scalp topography between 200-300 ms was similar, and in all conditions EB was aware of the presentation of one stimulus item. In contrast, the electrophysiological differences between illusory conjunction and extinction fall into the time period of preconscious vision (105-160 ms). We suggest that this early window reflects the moment in time when during competition the representation of the left color prevails over the right color. Support for such an early feature selection process comes from studies showing that focused attention affects early ERP components originating in extrastriate visual cortex, where only elementary features are represented (Hillyard and Anllo-Vento, 1998, Di Russo et al., 2001, Fellrath et al., 2014). P1, an ERP component showing amplitude modulation due to spatial shifts of attention is recorded between 70 and 130 ms after stimulus onset, which corresponds to the period of the first time window identified in our study. While focusing attention may simply amplify signals in early vision (<100 ms) its later effects (>200 ms) are more complex and suggest the involvement in distracter filtering, target detection and feature conjunction (Luck et al., 2000). The two time periods observed in our data support a twostage model of binding (similar to that suggested by Humphreys et al., 2000), in which preattentive features are first selected based on visual grouping and spatial competition and then bound into coherent objects by a capacity limited process.

In conclusion, the present results indicate that the time-point when a feature becomes part of a coherent, individuated object is much earlier than suggested by previous work. This finding is in agreement with earlier psychophysical (Houck and Hoffman, 1986, Holcombe and Cavanagh, 2001) and electrophysiological findings (Winkler et al., 2005, Muller et al., 2010) showing that feature conjunctions can be formed without attention. Thus, binding has to do with the focalization of attentional resources, but also relies on a preselection process that is activated very early in preattentive vision. These data question the utility of the term 'preattentive', as they suggest that selection processes operate already at an early level of visual processing (Hillyard and Anllo-Vento, 1998, Luck et al., 2000, Wolfe and Horowitz, 2004). A failure of such early selection processes may explain why patients with Bálint's syndrome perceive the world as a constant fusion and disjunction of seemingly freely floating features.

Acknowledgements: Study supported by the Swiss National Science Foundation (grant 320030-152689).

\section{REFERENCES}

Bálint R (1909) Seelenlähmung des 'Schauens', optische Ataxie, räumliche Störung der Aufmerksamkeit. Msschr Psychiat Neurol 25:51-66. 
Bernstein LJ, Robertson LC (1998) Illusory conjunctions of color and motion with shape following bilateral parietal lesions. Psych Sci 9:167-175.

Billock VA, Tsou BH (2004) What do catastrophic visual binding failures look like? Trends Neurosci 27:84-89.

Brunet D, Murray MM, Michel CM (2011) Spatiotemporal analysis of multichannel EEG: CARTOOL. Computational intelligence and neuroscience 2011:813870.

Cave KR, Bichot NP (1999) Visuospatial attention: beyond a spotlight model. Psychonom Bull Rev 6:204-223.

Cinel C, Humphreys GW (2006) On the relations between implicit and explicit spatial binding: evidence from Balint's syndrome. Cogn Affect Behav Neurosci 6:127-140.

Cohen A, Rafal RD (1991) Attention and feature integration: illusory conjunctions in a patient with a parietal lobe lesion. Psych Sci 2:106-110.

Corbetta M, Shulman GL, Miezin FM, Petersen SE (1995) Superior parietal cortex activation during spatial attention shifts and visual feature conjunction. Science 270:802-805.

Desimone R, Duncan J (1995) Neural mechanisms of selective visual attention. Ann Rev Neurosci 18:193-222.

Di Russo F, Martinez A, Sereno MI, Pitzalis S, Hillyard SA (2001) Cortical sources of the early components of the visual evoked potential. Human Brain Mapping 15:95-111.

Duncan J, Humphreys GW (1989) Visual search and stimulus similarity. Psychol Rev 96:433458.

Eckstein MP (2011) Visual search: a retrospective. J Vis 11.

Egner T, Monti JM, Trittschuh EH, Wieneke CA, Hirsch J, Mesulam MM (2008) Neural integration of top-down spatial and feature-based information in visual search. $J$ Neurosci 28:6141-6151.

Eimer M, Kiss M (2010) Top-down search strategies determine attentional capture in visual search: behavioral and electrophysiological evidence. Atten Percept Psychophys 72:951-962.

Felleman DJ, Van Essen DC (1991) Distributed hierarchical processing in the primate cortex. Cereb Cortex 1:1-47.

Fellrath J, Manuel AL, Ptak R (2014) Task relevance effects in electrophysiological brain activity: Early, but not first. Neurolmage 101:68-75.

Friedman-Hill SR, Robertson LC, Treisman A (1995) Parietal contributions to visual feature binding: Evidence from a patient with bilateral lesions. Science 269:853-855.

Gauthier L, Dehaut F, Joanette Y (1989) The Bells Test: A quantative and qualitative test for visual neglect. Int J Clin Neuropsychol 11:49-54.

Hickey C, Di Lollo V, McDonald JJ (2009) Electrophysiological indices of target and distractor processing in visual search. J Cogn Neurosci 21:760-775. 
Hillyard SA, Anllo-Vento L (1998) Event-related brain potentials in the study of visual selective attention. Proc Natl Acad Sci U S A 95:781-787.

Holcombe AO, Cavanagh P (2001) Early binding of feature pairs for visual perception. Nat Neurosci 4:127-128.

Houck MR, Hoffman JE (1986) Conjunction of color and form without attention: evidence from an orientation-contingent color aftereffect. J Exp Psychol Hum Percept Perform 12:186-199.

Humphreys GW, Cinel C, Wolfe J, Olson A, Klempen N (2000) Fractionating the binding process: neuropsychological evidence distinguishing binding of form from binding of surface features. Vision Res 40:1569-1596.

Koivisto M, Revonsuo A (2010) Event-related brain potential correlates of visual awareness. Neurosci Biobehav Rev 34:922-934.

Leonards U, Palix J, Michel C, Ibanez V (2003) Comparison of early cortical networks in efficient and inefficient visual search: an event-related potential study. J Cogn Neurosci 15:1039-1051.

Luck S, Fan S, Hillyard S (1993) Attention-related modulation of sensory-evoked brain activity in a visual search task. J Cogn Neurosci 5:188-195.

Luck SJ, Hillyard SA (1994) Spatial filtering during visual search: evidence from human electrophysiology. J Exp Psychol Hum Percept Perform 20:1000-1014.

Luck SJ, Woodman GF, Vogel EK (2000) Event-related potential studies of attention. Trends Cogn Sci 4:432-440.

Maris E, Oostenveld R (2007) Nonparametric statistical testing of EEG- and MEG-data. J Neurosci Methods 164:177-190.

Mitko A, Prinzmetal W, Esterman M, List A (2015) An EEG study of illusory conjunctions. Journal of Vision 15:894.

Muller D, Winkler I, Roeber U, Schaffer S, Czigler I, Schroger E (2010) Visual object representations can be formed outside the focus of voluntary attention: evidence from event-related brain potentials. J Cogn Neurosci 22:1179-1188.

Oostenveld R, Fries P, Maris E, Schoffelen JM (2011) FieldTrip: Open source software for advanced analysis of MEG, EEG, and invasive electrophysiological data. Comput Intell Neurosci 2011:156869.

Prinzmetal W, Henderson D, Ivry R (1995) Loosening the constraints on illusory conjunctions: assessing the roles of exposure duration and attention. J Exp Psychol Hum Percept Perform 21:1362-1375.

Ptak R, Fellrath J (2013) Spatial neglect and the neural coding of attentional priority. Neurosci Biobehav Rev 37:705-722. 
Ptak R, Lazeyras F, Di Pietro M, Schnider A, Simon SR (2014) Visual object agnosia is associated with a breakdown of object-selective responses in the lateral occipital cortex. Neuropsychologia 60:10-20.

Ptak R, Müri RM (2013) The parietal cortex and saccade planning: lessons from human lesion studies. Frontiers in Human Neuroscience 7:254.

Ptak R, Schnider A (2005) Visual extinction of similar and dissimilar stimuli: Evidence for level-dependent attentional competition. Cogn Neuropsychol 22:111-127.

Quinlan PT (2003) Visual feature integration theory: past, present, and future. Psychol Bull 129:643-673.

Rafal RD (1997) Balint syndrome. In: Behavioral Neurology and Neuropsychology (Feinberg, T. E. and Farah, M. J., eds), pp 337-356 New York: McGraw-Hill.

Reynolds JH, Desimone R (1999) The role of neural mechanisms of attention in solving the binding problem. Neuron 24:19-29.

Riddoch MJ, Humphreys GW (1993) BORB. Birmingham Object Recognition Battery. Hove: Lawrence Erlbaum Associates.

Robertson LC (2003) Binding, spatial attention and perceptual awareness. Nat Rev Neurosci 4:93-102.

Robertson LC, Treisman A, Friedman-Hill S, Grabowecky M (1997) The interaction of spatial and object pathways: evidence from Balint's syndrome. J Cogn Neurosci 9:295-317.

Shafritz KM, Gore JC, Marois R (2002) The role of the parietal cortex in visual feature binding. Proc Natl Acad Sci U S A 99:10917-10922.

Treisman A (1996) The binding problem. Curr Opin Neurobiol 6:171-178.

Treisman A (1998) Feature binding, attention and object perception. Phil Trans R Soc Lond B 353:1295-1306.

Treisman A, Gelade G (1980) A feature-integration theory of attention. Cogn Psychol 12:97136.

Treisman A, Gormican S (1988) Feature analysis in early vision: Evidence from search asymmetries. Psychol Rev 95:15-48.

Treisman A, Schmidt H (1982) Illusory conjunctions in the perception of objects. Cogn Psychol 14:107-141.

Valenza N, Murray MM, Ptak R, Vuilleumier P (2004) The space of senses: impaired crossmodal interactions in a patient with Balint syndrome after bilateral parietal damage. Neuropsychologia 42:1737-1748.

von der Malsburg C (1995) Binding in models of perception and brain function. Curr Opin Neurobiol 5:520-526.

Warrington EK, James M (1991) The Visual Object and Space Perception Battery. Bury St Edmunds: Thames Valley Test Company. 
Winkler I, Czigler I, Sussman E, Horvath J, Balazs L (2005) Preattentive binding of auditory and visual stimulus features. J Cogn Neurosci 17:320-339.

Wolfe JM (1994) Guided Search 2.0. A revised model of visual search. Psychonom Bull Rev $1: 202-238$.

Wolfe JM, Bennett SC (1997) Preattentive object files: shapeless bundles of basic features. Vision Res 37:25-43.

Wolfe JM, Cave KR (1999) The psychophysical evidence for a binding problem in human vision. Neuron 24:11-17, 111-125.

Wolfe JM, Horowitz TS (2004) What attributes guide the deployment of visual attention and how do they do it? Nat Rev Neurosci 5:1-7. 
Table 1. Performance of EB in tests probing visual-spatial functions.

\begin{tabular}{|c|c|c|}
\hline Test & Score & $\begin{array}{c}\text { Deficit } \\
\text { classification }\end{array}$ \\
\hline \multicolumn{3}{|c|}{ Birmingham Object Recognition Battery $^{1}$} \\
\hline Size match & $4 / 10$ & severe \\
\hline Length match & $13 / 30$ & severe \\
\hline \multicolumn{3}{|c|}{ Visual Object and Space Perception Battery ${ }^{2}$} \\
\hline Incomplete letters & $0 / 20$ & severe \\
\hline Silhouettes & $7 / 20$ & moderate \\
\hline Object decision & $10 / 20$ & moderate \\
\hline Progressive silhouettes & $14 / 20$ & preserved \\
\hline Dot counting & $1 / 10$ & severe \\
\hline Position discrimination & $9 / 20$ & severe \\
\hline Number location & $0 / 10$ & severe \\
\hline Cube analysis & $4 / 10$ & severe \\
\hline \multicolumn{3}{|l|}{ Bells cancellation ${ }^{3}$} \\
\hline Left omissions & 7 & severe \\
\hline Right omissions & 8 & severe \\
\hline Naming (real object) ${ }^{4}$ & $27 / 35$ & moderate \\
\hline Naming (line drawing) ${ }^{4}$ & $18 / 35$ & severe \\
\hline Letter identification & $21 / 23$ & moderate \\
\hline
\end{tabular}

${ }^{1}$ (Riddoch and Humphreys, 1993); ${ }^{2}$ (Warrington and James, 1991); ${ }^{3}$ (Gauthier et al., 1989); ${ }^{4}$ (Ptak et al., 2014) 


\section{Figure legends}

Figure 1. A Left, posterior and right view of EB's brain showing bilateral parietal damage, while the occipital cortex appears preserved. B Examples of double simultaneous displays presented in the three experiments. Note that letters are not shown in the original scale and that in reality all stimuli were either white or colored and shown on black background.

Figure 2. A Identification and detection of letters presented left and right (Experiment 1), or up and down (Experiment 2) of the fixation cross. Dark blue bars indicate trials when EB was able to identify the letter while light blue shows additional trials on which EB detected 'something' without being able to name the letter. B Frequency of mislocalized letters in horizontal or vertical presentation. The dashed horizontal line indicates chance performance (L: left; R: right; D: down; U: up).

Figure 3. A Identification of letter shape, color, or both features as a function of hemifield and presentation in single or double displays. B Localization errors of correctly identified features.

Figure 4. Scalp topographies of the four experimental conditions for three time windows. The nose is shown up and the left side of the scalp is shown on the left. The mapping of the electrode arrangement to the $10-20$ system is shown on the left.

Figure 5. Comparison of ERPs averaged over a right anterior cluster of electrodes (the cluster identified with permutation testing is highlighted in the topographic plot), as a function of experimental condition (red: illusory conjunction of right shape with left color; blue: extinction of the left item). The scalp topography shows the difference in voltages between conjunction and extinction conditions, averaged over the time window 105-160 ms (shown as grey area in the ERP plot).

Figure 6. ERP data obtained at anterior and posterior electrodes, as a function of experimental condition (red: illusory conjunction; blue: extinction). The grey area shows the period of significant difference in permutation testing. 


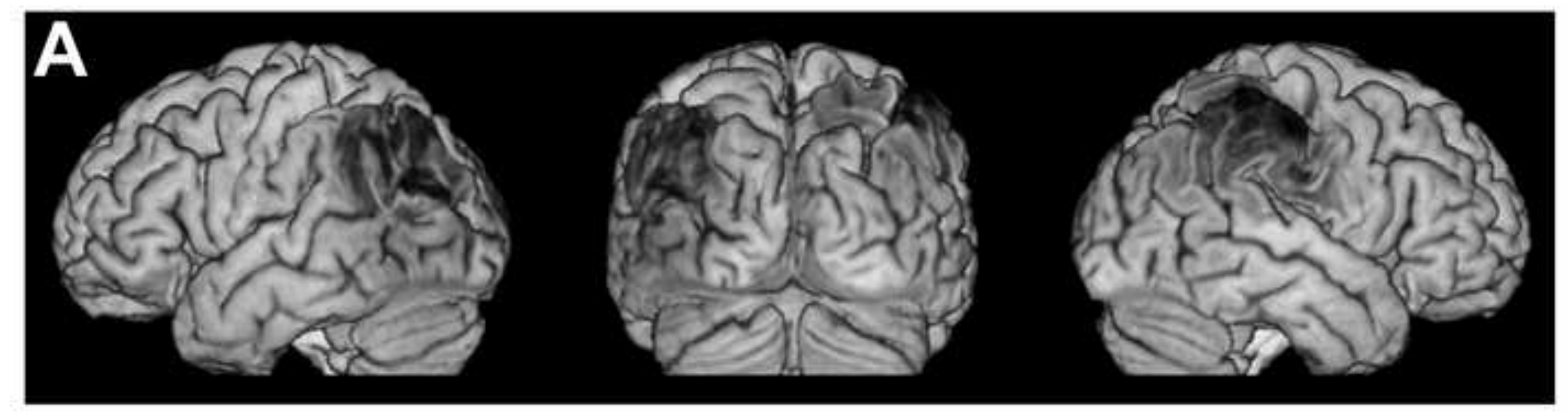

\section{B Experiment 1}

Experiment 2

Experiment 3
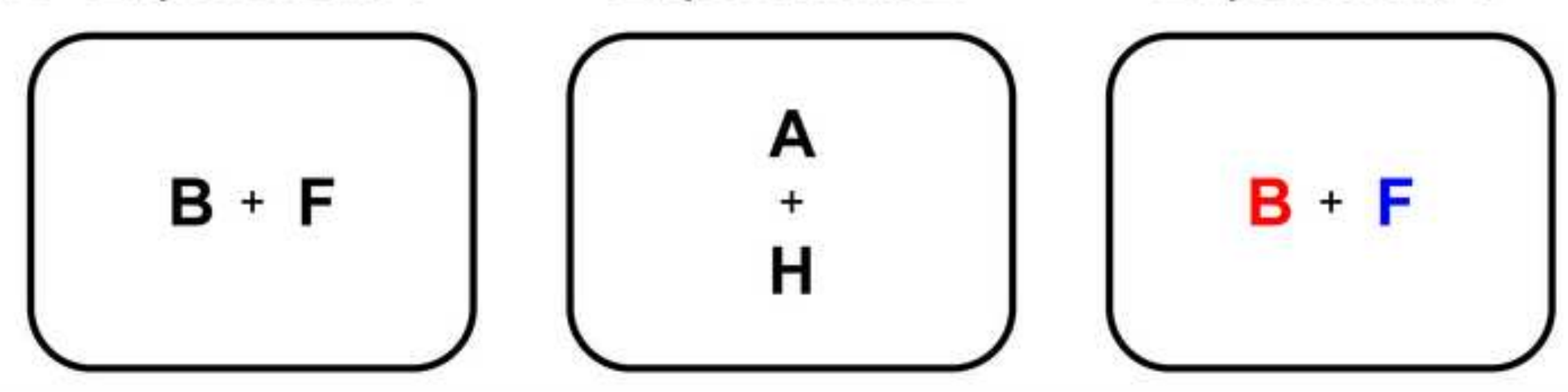

$$
B+F
$$

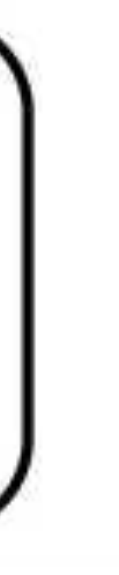



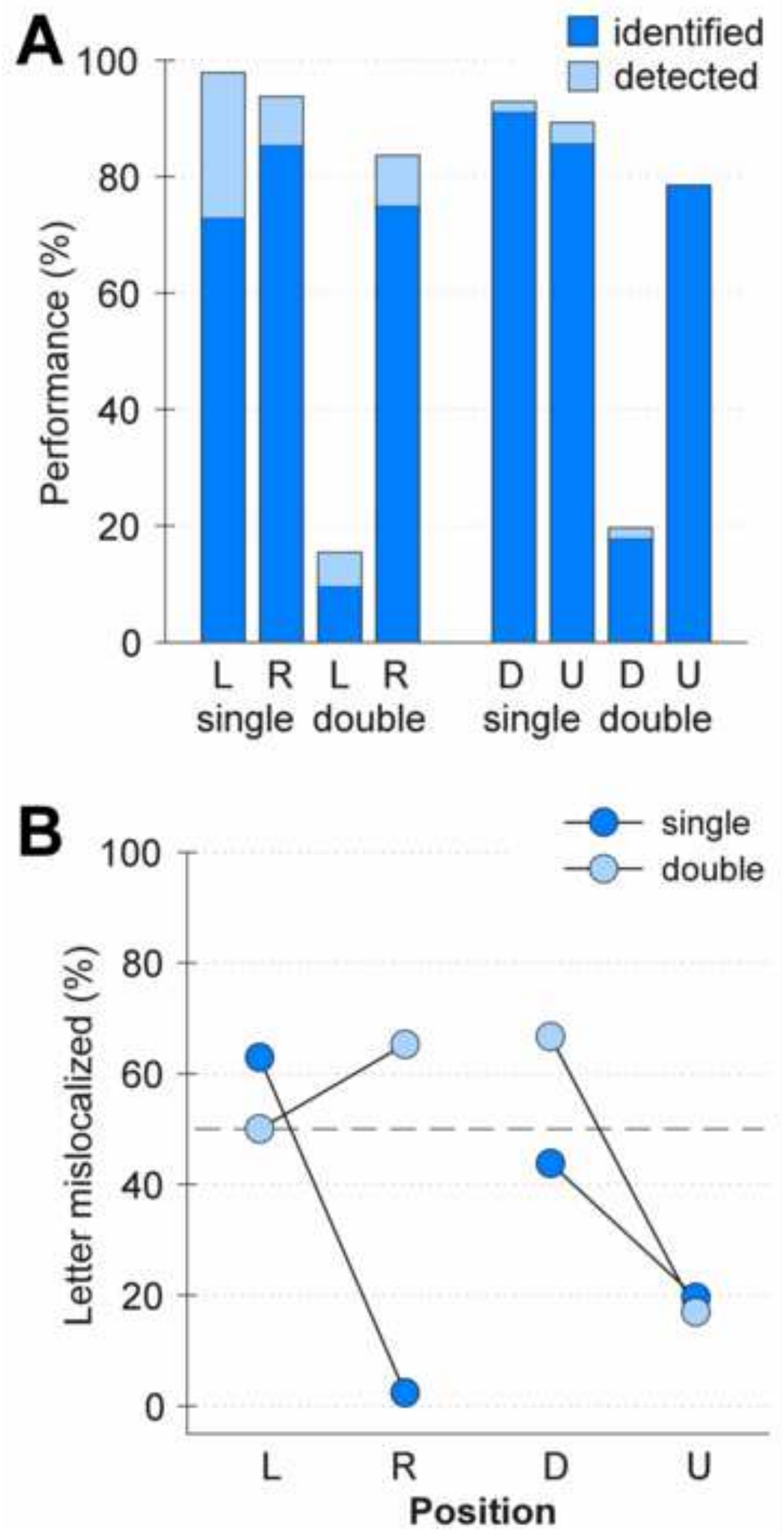

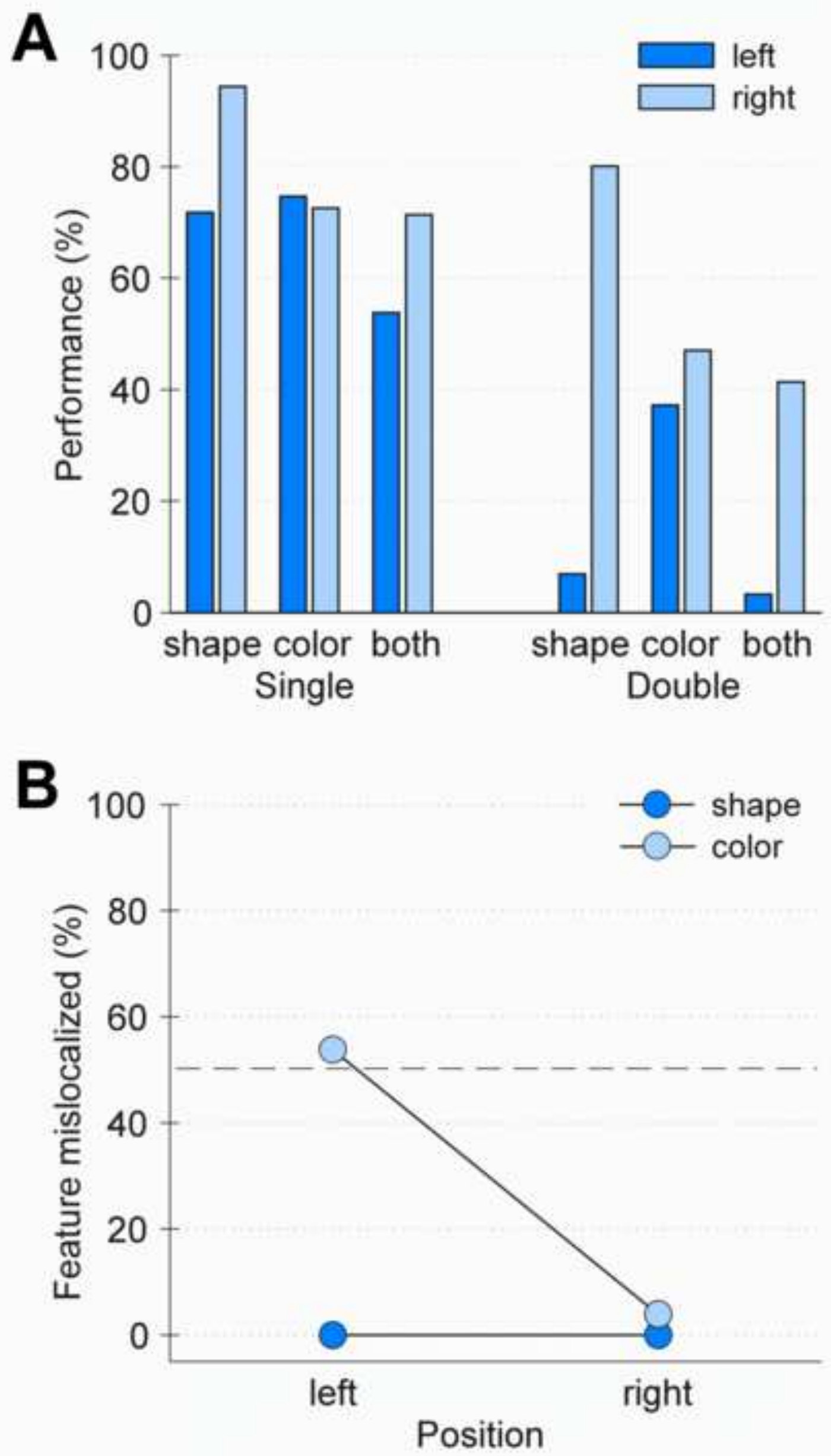


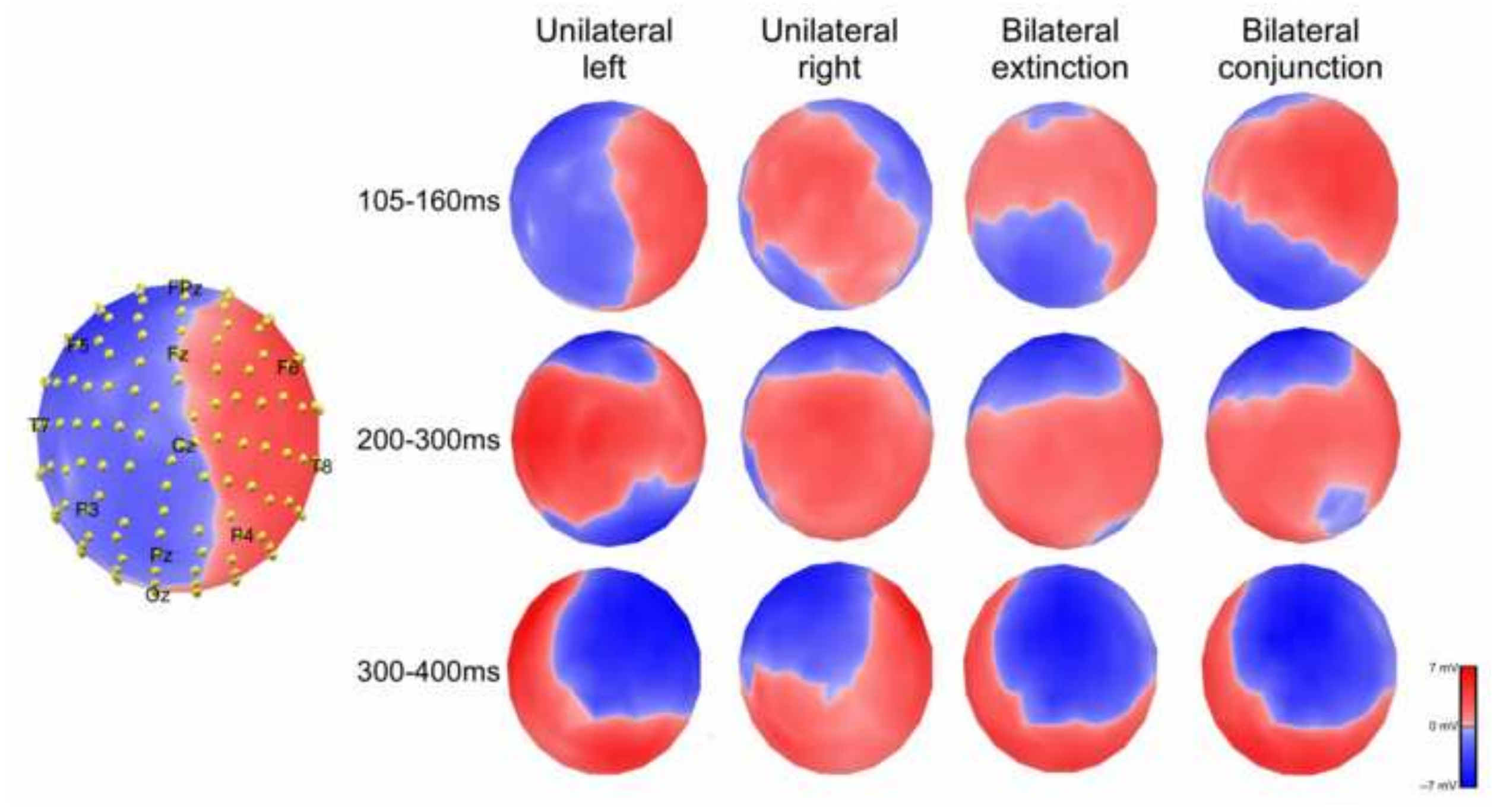




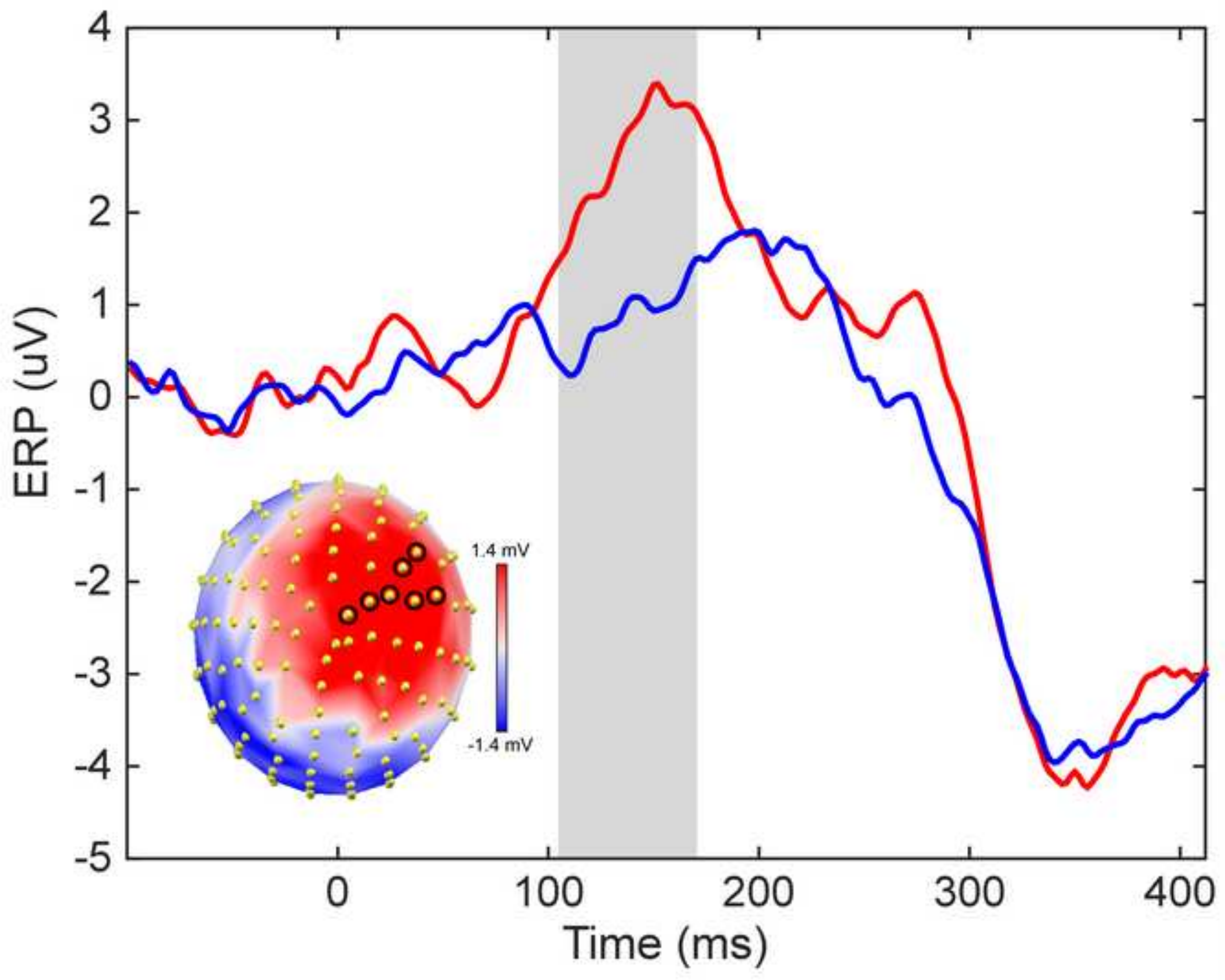



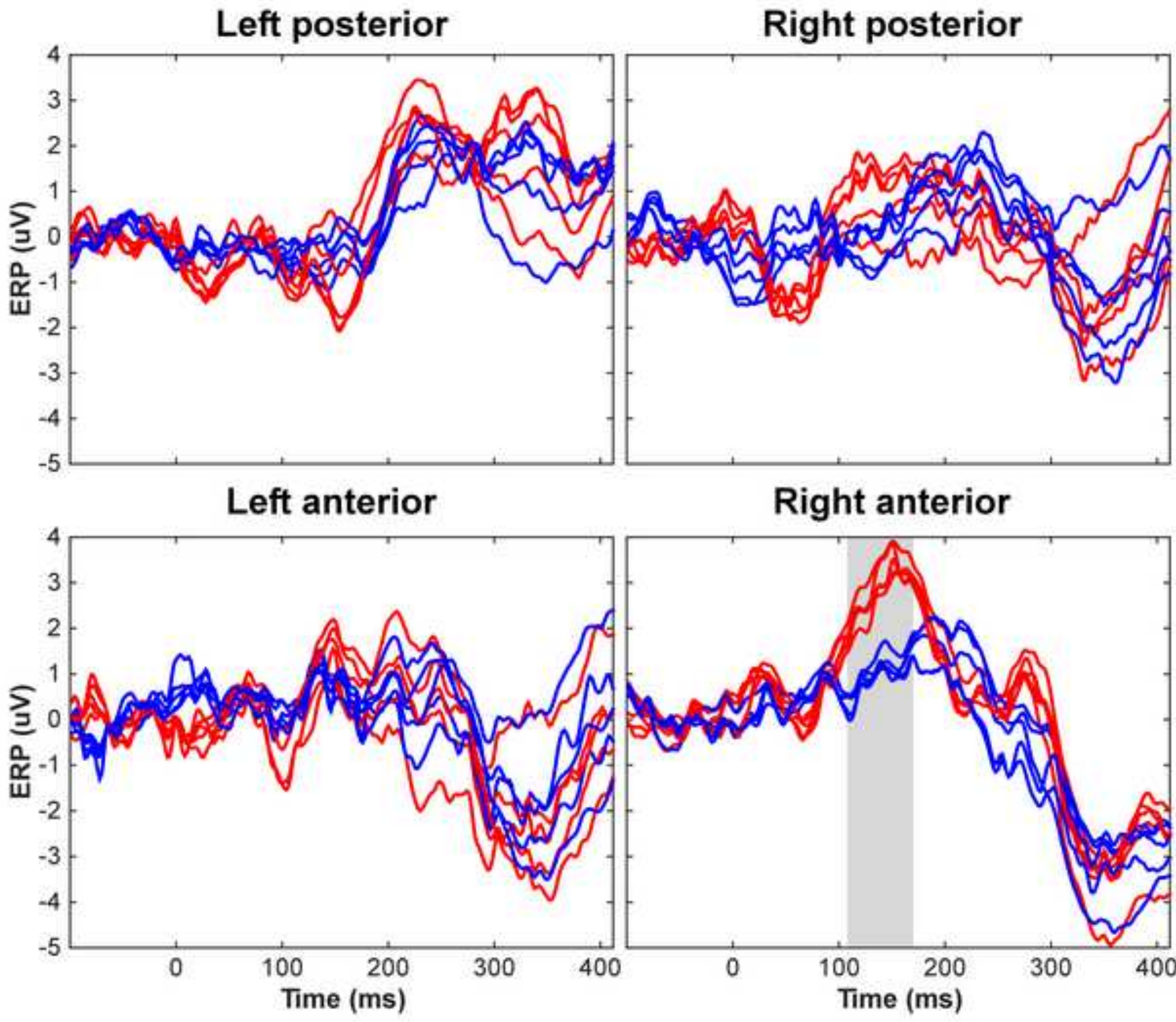

Right anterior

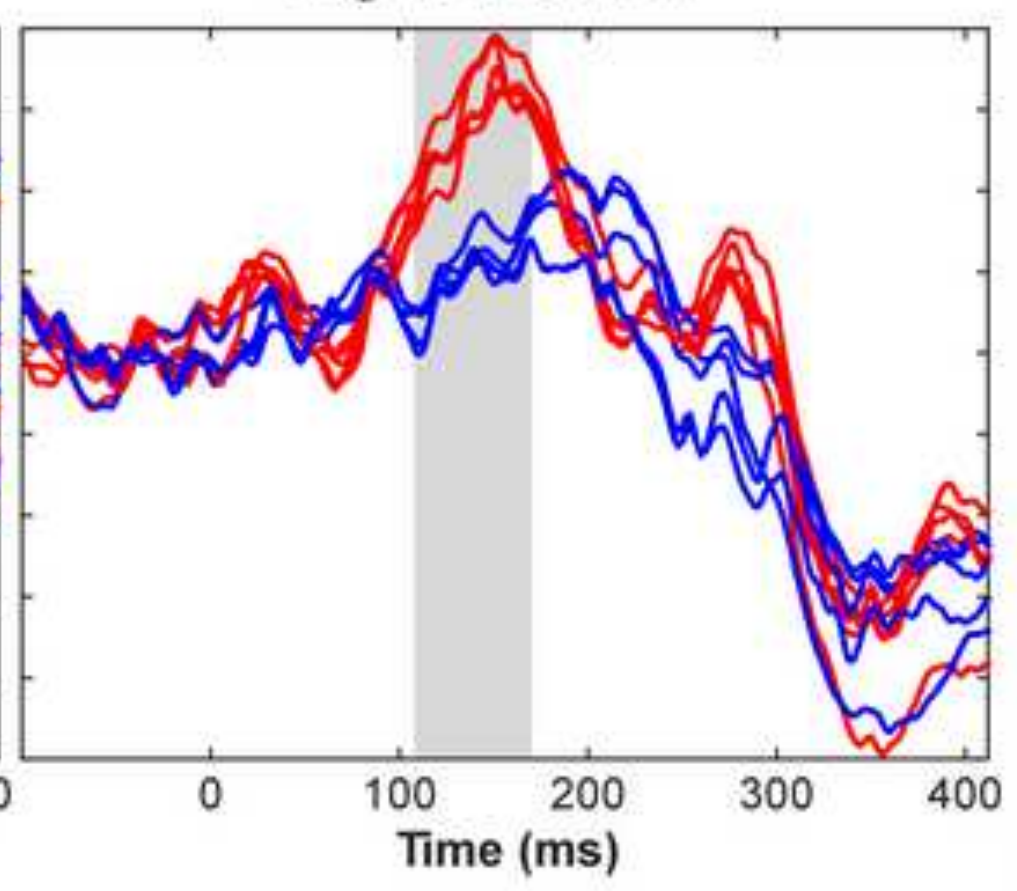

